\section{Relación entre el capital social y el consumo de alcohol, tabaco y drogas}

Algunas teorías propias de las ciencias sociales pueden ser de interés para los profesionales de la salud pública. La teoría del capital social, por ejemplo, propone que el patrimonio intrínseco de las relaciones sociales puede contribuir a los resultados que alcanzan las personas en la vida, por ende los jóvenes con mayor capital social $-\mathrm{o}$ la ventaja aportada por las relaciones sociales - tendrían menos probabilidades de confrontar dificultades sociales y conductuales. De esta forma, el capital social ha ganado un lugar en la investigación educacional en los últimos años y puede ser útil en la investigación sanitaria. Como concepto analítico es multidimensional y está compuesto por cuatro elementos: las reglas y expectativas de los padres, el clima familiar, la conexión intrafamiliar y el capital humano. Como propiedad que depende de las relaciones interpersonales, el capital social se expresa en redes recíprocas de cooperación y confianza, en las que se comparten normas comunes. Estas redes aportan ventajas a los individuos.

El capital social ha sido objeto de estudio en diversos campos académicos, particularmente en la investigación educacional. El análisis de las tendencias actuales puede aportar una valiosa información sobre la manera de fortalecer los resultados individuales y colectivos y contribuir al desarrollo de adultos saludables, exitosos y productivos.

En este trabajo se analiza la relación que existe entre el capital social y el consumo de alcohol, tabaco y drogas por adolescentes y se identifican los factores del capital social que influyen en la prevención de conductas adictivas.

Para ello se realizó un análisis secundario de las respuestas de 590 estudiantes de la enseñanza media, de 14 años o más, a la Encuesta sobre Factores Protectores y de Riesgo en Jóvenes, realizada en la primavera de 2003 en una ciudad del medio oeste de los Estados Unidos de América. En esta encuesta de 102 preguntas con respuestas múltiples se recabó información sobre los factores protectores y de riesgo, los comportamientos de alto riesgo (consumo de alcohol, tabaco, marihuana y otras drogas) e indicadores de capital social.

En este estudio se encontraron diversas asociaciones significativas. Entre el consumo de alcohol y dos factores de capital social (las reglas y expectativas de los padres y la conexión intrafamiliar); el consumo de alcohol en la escuela y tres factores (las reglas y expectativas de los padres, el clima familiar y el capital humano); el consumo de tabaco y los cuatro factores (las reglas y expectativas de los padres, el capital humano, el clima familiar y la conexión intrafamiliar); el consumo de marihuana y un factor (las reglas y expectativas de los padres); y el consumo de otras drogas y tres factores (las reglas y expectativas de los padres, el clima familiar y el capital humano).

El resultado de este análisis demostró que existe una relación global entre el capital social y el consumo de alcohol, tabaco y drogas en el conjunto de jóvenes estudiados. En general, cuanto mayor es el capital humano menor es la probabilidad de que el adolescente asuma conductas de alto riesgo. El capital humano y el clima familiar estuvieron consistentemente asociados con el consumo de tabaco y drogas en cada uno de los modelos, pero siempre en menor medida que las reglas y las expectativas de los padres.

Estas relaciones intuitivas y lógicas reciben ahora un respaldo teórico descrito como la contribución del capital social a la mejor evolución, tanto de los individuos aislados como de los grupos de individuos. Los adolescentes deben contar con todas las oportunidades para desarrollar y aplicar los recursos de su capital humano. Se debe hacer un esfuerzo adicional para ayudar a los adolescentes a desarrollar habilidades de comunicación que les permitan discutir cuestiones relacionadas con el clima familiar con personas capacitadas y confiables y encontrar los recursos que necesitan.

Aunque la contribución de la conexión intrafamiliar con los factores relacionados con el consumo de alcohol, tabaco y drogas se deben interpretar con gran cautela debido a la inestabilidad e incertidumbre que rodea la medición de la conexión intrafamiliar, estos resultados son importantes y se pueden aplicar a investigaciones de interés para la salud pública. (Curran EM. The relationship between social capital and substance use by high school students. J Alcohol Drug Educ. 2007; junio. Hallado en http:/ / www.thefreelibrary.com/ The+relationship+between+social+capital+and +substance+use+by+high . . . -a0165912681. Acceso el 22 de agosto de 2007.) 